\title{
Practical Risk Prediction Tools for Coronary Heart Disease in Mild to Moderate Hypercholesterolemia in Japan Originated From the MEGA Study Data
}

\author{
Tamio Teramoto, MD; Yasuo Ohashi, PhD*; Noriaki Nakaya, MD**; \\ Shinji Yokoyama, $\mathrm{MD}^{\dagger}$; Kyoichi Mizuno, MD ${ }^{\dagger}$; Haruo Nakamura, MD \\ for the MEGA Study Group
}

\begin{abstract}
Background A simple and practical risk prediction tool for coronary heart disease (CHD) to determine the specific risk level in each patient that fits the true clinical practice setting is needed and would be valuable in Japan.

Methods and Results A 5-year risk prediction score and chart for CHD based on the MEGA study data was developed in the present study. The MEGA risk prediction score and chart were constructed based on the coefficient of each risk factor. The risk factors included in these risk prediction tools were: treatment (diet, diet plus pravastatin), sex, age, baseline high-density lipoprotein-cholesterol, baseline low-density lipoprotein-cholesterol, glucose abnormality (diabetes and impaired fasting glucose), hypertension, and smoking. The MEGA risk prediction score comprised the risk score for each risk factor, and it can predict 5-year risk for CHD with 5 levels of risk, based on the total risk score. The MEGA risk prediction chart more accurately predicts risk, by reflecting the accumulation of risk factors and using an 8-color visual chart.

Conclusions The MEGA risk prediction score and chart, developed from the MEGA study data, more easily and accurately assesses the 5-year CHD risk in mild to moderate hypercholesterolemic patients in the usual clinical practice setting in Japan. (Circ J 2008; 72: 1569-1575)
\end{abstract}

Key Words: Coronary artery disease; Follow-up studies; Hypercholesterolemia; Risk factors; Statin

C oronary heart disease (CHD) represents one of the main causes of death in the USA and Europe, and is the second most frequent cause of death in Japan? CHD risk increases remarkably with the accumulation of risk factors ${ }^{3-5}$ Data from some epidemiological studies and clinical trials have identified several risk factors for $\mathrm{CHD}^{6,7}$ and some risk prediction scores for $\mathrm{CHD}$ were developed using risk factor analysis from these data. The Framingham risk model is a typical one? However, it is well known that the estimated incidence of CHD by the Framingham prediction model is not consistent with the actual incidence in different populations. Therefore, different prediction scores have been developed in several countries $^{9-12}$ In 2006, the Health Risk Evaluation Chart ${ }^{13}$ a risk chart corresponding to the Framingham CHD risk score, was developed based on the Nippon Data $80,{ }^{14}$ which used a 19-year follow-up study of data of the Japanese general population.

(Received March 6, 2008; revised manuscript received May 29, 2008; accepted June 11, 2008; released online September 2, 2008)

Department of Internal Medicine, Teikyo University School of Medicine, *Department of Biostatistics/Epidemiology and Preventive Health Sciences, The University of Tokyo, **Nakaya Clinic, Tokyo, Biochemistry, Cell Biology and Metabolism, Nagoya City University Graduate School of Medical Sciences, Nagoya, ${ }^{+1}$ Department of Medicine, Nippon Medical School and $¥$ Mitsukoshi Health and Welfare Foundation, Tokyo, Japan

Mailing address: Tamio Teramoto, MD, Department of Internal Medicine, Teikyo University School of Medicine, 2-11-1 Kaga, Itabashi-ku, Tokyo 173-8605, Japan. E-mail: ttera@med.teikyo-u.ac.jp All rights are reserved to the Japanese Circulation Society. For permissions, please e-mail: cj@j-circ.or.jp
Thus, despite the different risk prediction tools available in several countries, it remains unclear whether these tools accurately predict risk in patients with hypercholesterolemia treated by diet with or without a statin. Regarding risk prediction for the population receiving lipid-lowering pharmacotherapy, the CHD predicted value obtained from the Framingham risk model was compared with the observed CHD incidence in a substudy of WOSCOPS 15 conducted to confirm the efficacy of pravastatin to prevent the first onset of ischemic heart disease. The observed incidence of CHD was similar to the predicted CHD risk using the Framingham risk model in the placebo group, whereas in the pravastatin group the observed CHD incidence was lower than the predicted CHD risk, indicating that the Framingham risk model does not accurately apply to patients receiving pravastatin! 16

The MEGA study is a large-scale clinical study conducted to evaluate the efficacy of pravastatin treatment to decrease the risk of cardiovascular events in patients with mild to moderate hypercholesterolemia without a past history of ischemic heart disease and/or stroke!7,18 This report shows that 2 different 5-year CHD incidence risk prediction tools, a risk prediction score and chart, developed from the MEGA study data is accurate and efficient for clinical application.

\section{Methods}

The MEGA study, a prospective randomized open-label study, was conducted from February 1994 to March 2004. A total of 8,214 patients with hypercholesterolemia (total cholesterol (TC) $220-270 \mathrm{mg} / \mathrm{dl}$ ) and no history of ischemic 
Table 1 Baseline Characteristics of Study Patients in the MEGA Study

\begin{tabular}{lcc}
\hline \hline & Diet group & Diet plus pravastatin group \\
\hline No. of patients & 3,966 & 3,866 \\
Age $($ years $)$ & $58.4 \pm 7.2$ & $58.2 \pm 7.3$ \\
Women & $2,718(69 \%)$ & $2,638(68 \%)$ \\
BMI $\left(\mathrm{kg} / \mathrm{m}^{2}\right)$ & $23.8 \pm 3.0$ & $23.8 \pm 3.1$ \\
SBP $(\mathrm{mmHg})$ & $132.4 \pm 16.8$ & $132.0 \pm 16.8$ \\
DBP $(\mathrm{mmHg})$ & $78.8 \pm 10.2$ & $78.4 \pm 10.4$ \\
HT* & $1,664(42 \%)$ & $1,613(42 \%)$ \\
Glucose abnormality** & $828(21 \%)$ & $804(21 \%)$ \\
Current/past smoker & $791(20 \%)$ & $823(21 \%)$ \\
TC $(\mathrm{mg} / \mathrm{dl})$ & $242.6 \pm 12.2$ & $242.6 \pm 12.1$ \\
TG $(\mathrm{mg} / \mathrm{dl})^{\dagger}$ & $127.5(37.0-1,322.5)$ & $127.4(34.5-1,010.0)$ \\
HDL-C $(\mathrm{mg} / \mathrm{dl})$ & $57.5 \pm 15.1$ & $57.5 \pm 15.0$ \\
LDL-C $(\mathrm{mg} / \mathrm{dl})$ & $156.5 \pm 17.6$ & $156.6 \pm 17.5$ \\
Lipoprotein $(\mathrm{a})(\mathrm{mg} / \mathrm{dl})$ & $24.7 \pm 25.2$ & $24.7 \pm 25.6$ \\
\hline
\end{tabular}

*Reported by physicians. **Documented diabetes and it also included the patients who had fasting glucose equal or greater than $110 \mathrm{mg} / \mathrm{dl}$ (impaired fasting glcose). ${ }^{\dagger}$ Data are median (interquartile range). All data are mean $\pm S D$ or number $(\%)$ unless otherwise indicated.

BMI, body mass index; SBP, systolic blood pressure; DBP, diastolic blood pressure; HT, hypertension; TC, total cholesterol; TG, triglyceride; HDL-C, high-density lipoprotein-cholesterol; LDL-C, low-density lipoprotein-cholesterol.

heart disease or stroke were enrolled. They comprised men of 40-70 years of age and post-menopausal women up to 70 years of age. The patients were assigned either to diet alone (diet group) or diet in combination with pravastatin treatment (10-20 mg/day, approved dose in Japan; diet plus pravastatin group). The mean follow-up period was 5.3 years. The primary endpoint was CHD (fatal and non-fatal myocardial infarction, angina pectoris, cardiac/sudden death, and angioplasty). Secondary endpoints were stroke, all cardiovascular disease, and total mortality. Major exclusion criteria included familial hypercholesterolemia, a history of cardiovascular disease, a current diagnosis of malignancy, and secondary hyperlipidemia.

Patients were evaluated, including the onset of endpoints, by the attending physician at 1,3 and 6 months after the start of follow-up and every 6 months thereafter.

For each event, the diagnosis was made by the attending physician (including data from electrocardiogram and myocardial scintigraphy as needed) and reported in detail. Electrocardiography was performed annually. Information on individual patients was entered in the case report forms by their attending physicians and reported to the central Data Center. The Endpoint Committee evaluated each event in a blinded manner according to the criteria we reported previously 17 Throughout the study period, TC, high-density lipoprotein-cholesterol (HDL-C), triglycerides (TG), and lipoprotein(a) $[\mathrm{Lp}(\mathrm{a})]$ concentrations were centrally measured at the same laboratory using methods standardized by the Centers for Disease Control and Prevention (CDC; Atlanta, GA). Low-density lipoprotein-cholesterol (LDL-C) concentration was estimated by the Friedewald formula 19 The intention-to-treat analysis comprised 7,832 patients. Details of the design of MEGA study and the main results were reported previously! ${ }^{7}$

To construct the risk prediction tools, 7,760 of the 7,832 patients were evaluated using explanatory variables (72 of the 7,832 patients were excluded because of missing explanatory variables). To determine the explanatory variables, a univariate analysis was performed and then the significant factors were incorporated into the multivariate analysis model. For the risk factors determined by this multivariate model, $\mathrm{p}<0.20$ served as the criterion for backward elimination of variables. The factors included in the tools were: treatment group (diet group, diet plus pravastatin group), sex (male, female), age ( $\leq 54,55-59,60-64, \geq 65$ years), baseline HDL-C $(<40,40-<60, \geq 60 \mathrm{mg} / \mathrm{dl})$, baseline LDL$\mathrm{C}(<140,140-<160, \geq 160 \mathrm{mg} / \mathrm{dl})$, glucose abnormality and hypertension (none, hypertension and normal fasting blood glucose concentration, glucose abnormality and normal blood pressure, glucose abnormality and hypertension), and smoking habit (non-smoker [including ex-smoker], smokers). Although baseline LDL-C was not identified as a risk factor for CHD in the present study, it was included in the MEGA risk tools because it was included in other risk prediction models. Because abnormality in glucose tolerance was included in glucose abnormality as a risk factor in the Guidelines for Arteriosclerosis 200720 patients with a fasting blood glucose concentration $\geq 110 \mathrm{mg} / \mathrm{dl}$ were included as diabetics in the present study.

To construct the MEGA risk prediction score, each risk score was established as an integer, taking into consideration the coefficient of each explanatory variable. The total risk score of each patient was calculated as a sum of the risk scores, and classified into 5 risk levels based on population quintiles for both treatment groups. The 5-year predicted value by each risk level was estimated from the Cox proportional hazard model to be used as the mean value of the predicted value at 5 years for each patient, and calculated for each treatment group? ${ }^{21}$ To confirm the precision of the MEGA risk prediction model, we visually compared the estimated value to the observed value, and we plotted the receiver operating characteristic (ROC) curve using the development of CHD as the endpoint, and predictability was compared using the area under the ROC curve.

Further, we developed a simple, 5-year risk prediction chart based on the coefficient of each explanatory variable. The MEGA 5-year risk assessment chart for CHD displays the lipid parameters (HDL-C, LDL-C) by age on the y-axis and the characteristics associated with CHD risk (sex, smoking, hypertension, glucose abnormality) on the $\mathrm{x}$-axis. In constructing the chart, the 2 categories of HDL-C $(<40$, $40-<60 \mathrm{mg} / \mathrm{dl})$ and LDL-C $(140-<160, \geq 160 \mathrm{mg} / \mathrm{dl})$ were integrated because the risk scores were set to the same degree. The 5-year CHD risk of each cell was estimated by the Cox proportional hazard model, and 8 levels of risk defined (each with its own color). The layout of the chart optimizes 
Table 2 The $\beta$-Coefficients and HRs of the Multivariable Cox Proportional Hazard Model for 5-Year Risk of Coronary Heart Disease

\begin{tabular}{|c|c|c|c|}
\hline & $\beta$ & $H R$ & $95 \% C I$ \\
\hline \multicolumn{4}{|l|}{ Groups } \\
\hline Diet group & 0 & 1.00 & _- \\
\hline Diet plus pravastatin group & -0.350 & 0.70 & $0.50-0.99$ \\
\hline \multicolumn{4}{|l|}{ Sex } \\
\hline Women & 0 & 1.00 & - \\
\hline Men & 0.784 & 2.19 & $1.49-3.21$ \\
\hline \multicolumn{4}{|l|}{ Age } \\
\hline$<55$ & 0 & 1.00 & - \\
\hline $55-59$ & 0.231 & 1.26 & $0.72-2.19$ \\
\hline $60-64$ & 0.566 & 1.76 & $1.07-2.89$ \\
\hline$\geq 65$ & 0.932 & 2.54 & $1.58-4.08$ \\
\hline \multicolumn{4}{|l|}{$H D L-C(m g / d l)$} \\
\hline$\geq 60$ & 0 & 1.00 & _ \\
\hline $40-<60$ & 0.714 & 2.04 & $1.29-3.24$ \\
\hline$<40$ & 0.683 & 1.98 & $1.05-3.74$ \\
\hline \multicolumn{4}{|l|}{$L D L-C(m g / d l)$} \\
\hline$<140$ & 0 & 1.00 & - \\
\hline $140-<160$ & 0.230 & 1.26 & $0.72-2.19$ \\
\hline$\geq 160$ & 0.274 & 1.32 & $0.76-2.29$ \\
\hline \multicolumn{4}{|l|}{$H T^{*}$ glucose abnormality $* *$} \\
\hline No & 0 & 1.00 & - \\
\hline HT and normal fasting glucose $e^{\dagger}$ & 1.125 & 3.08 & $1.71-5.55$ \\
\hline Glucose abnormality and normal BP & 1.646 & 5.19 & $2.88-9.35$ \\
\hline Glucose abnormality and $H T$ & 1.992 & 7.33 & $4.12-13.04$ \\
\hline \multicolumn{4}{|l|}{ Current smoker } \\
\hline No & 0 & 1.00 & - \\
\hline Yes & 0.409 & 1.51 & $0.99-2.28$ \\
\hline
\end{tabular}

*Documented. **Documented diabetes and it also included the patients who had fasting glucose equal or greater than $110 \mathrm{mg} / \mathrm{dl}$ (impaired fasting glcose). ${ }^{\dagger}$ Normal fasting glucose was defined as patients meet following criteria; reported as non-diabetes by physicians and fasting plasma glucose less than $110 \mathrm{mg} / \mathrm{dl}$. ${ }^{\dagger}$ Normal BP was defind as patients reported as non-HT by physicians. HR, hazard ratio; CI, confidence interval; BP, blood pressure. Other abbreviations see in Table 1.

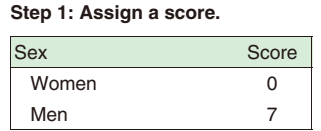

\begin{tabular}{|lc|}
\hline HDL-C $(\mathrm{mg} / \mathrm{dl})$ & Score \\
\hline$\geq 60$ & 0 \\
$40-<60$ & 6 \\
$<40$ & 6 \\
\hline
\end{tabular}

\begin{tabular}{|lc|}
\hline Age & Score \\
\hline$<55$ & 0 \\
$55-59$ & 2 \\
$60-64$ & 5 \\
$\geq 65$ & 8 \\
\hline
\end{tabular}

\begin{tabular}{|lc|}
\hline LDL-C $(\mathrm{mg} / \mathrm{dl})$ & Score \\
\hline$<140$ & 0 \\
$140-<160$ & 2 \\
$\geq 160$ & 2 \\
\hline
\end{tabular}

\begin{tabular}{|c|c|}
\hline \multicolumn{2}{|c|}{ Glucose abnormality, Hypertension } \\
\hline \multicolumn{2}{|l|}{ No } \\
\hline \multicolumn{2}{|c|}{ Hypertension and normal fasting } \\
\hline \multicolumn{2}{|c|}{ Glucose abnormality and normal } \\
\hline \multicolumn{2}{|c|}{ Glucose abnormality and hyperte } \\
\hline Current smoker & Score \\
\hline No & 0 \\
\hline Yes & 3 \\
\hline
\end{tabular}

Step 2: Add sum of scores.
\begin{tabular}{|lc|}
\hline Risk factor & Risk score \\
\hline Sex & $a$ \\
Age & $b$ \\
HDL-C & $c$ \\
LDL-C & $d$ \\
Glucose abnormality, Hypertension & $e$ \\
Smoking & $f$ \\
& \\
Total risk score & sum (a to f) \\
\hline
\end{tabular}

Step 3: Find absolute risk according to treatment.

\begin{tabular}{|lcc|}
\hline \multicolumn{1}{|c|}{$\begin{array}{c}\text { Total } \\
\text { risk score }\end{array}$} & 5-year CHD risk (\%) \\
\hline$<10$ & 0.3 & Diet plus pravastatin group \\
$10-15$ & 0.6 & 0.2 \\
$16-21$ & 1.2 & 0.4 \\
$22-26$ & 2.5 & 0.9 \\
$\geq 27$ & 6.4 & 1.8 \\
\hline
\end{tabular}

Fig 1. Simplified calculation form for estimating the 5-year risk of coronary heart disease (CHD) incidence in the diet group and diet plus pravastatin group. HDL-C, high-density lipoprotein-cholesterol: LDL-C, low-density lipoprotein-cholesterol.

the visual expression of the person's risk level from low to high with an 8-color gradation from the lower-left to the upper-right. For statistical analyses, the SAS software (release 8.2, SAS Institute, Cary, NC, USA) was used.

\section{Results}

During the 5-year follow-up, $138 \mathrm{CHD}$ events were ob- served in the MEGA study. The baseline characteristics of the study population are shown in Table 1 . The coefficient of each risk factor for CHD obtained from the Cox proportional hazard model and hazard ratio are shown in Table 2, and the 5-year risk prediction processes using the MEGA risk prediction score for CHD are summarized in Fig 1. A proportionally higher risk score was found for glucose abnormality alone (risk score 14), hypertension alone (risk 
Table 3 Baseline Characteristics in Each Risk Level

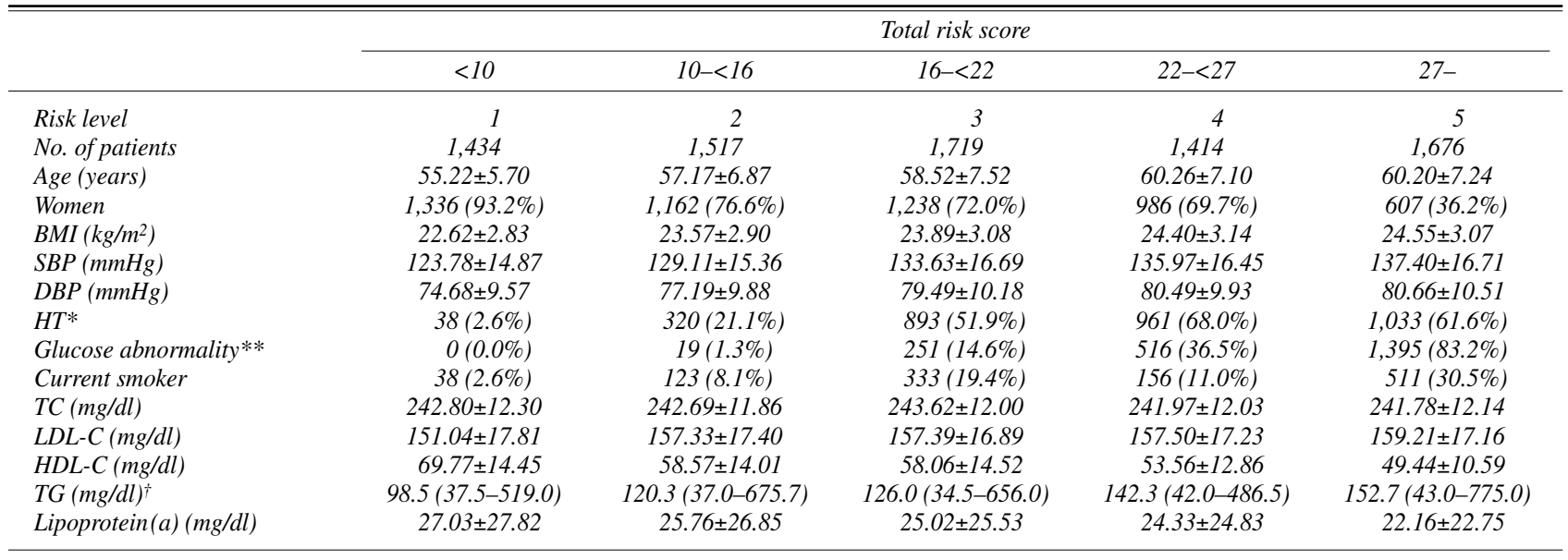

*Reported by physicians. **Documented diabetes and it also included the patients who had fasting glucose equal or greater than $110 \mathrm{mg} / \mathrm{dl}$ (impaired fasting glucose). ${ }^{\top}$ Data are median (interquartile range). All data are mean $\pm S D$ or number $(\%)$ unless otherwise indicated. The 72 patients were excluded from this analysis because of missing data of the risk factors for coronary heart disease.
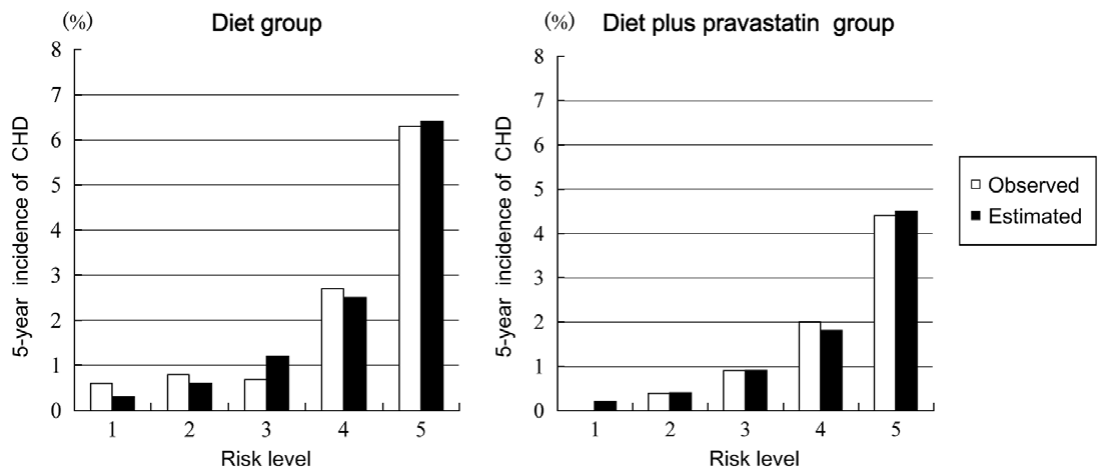

Fig 2. Comparison between the observed and estimated 5-year incidence of coronary heart disease (CHD) for the diet group and the diet plus pravastatin group.

Diet group

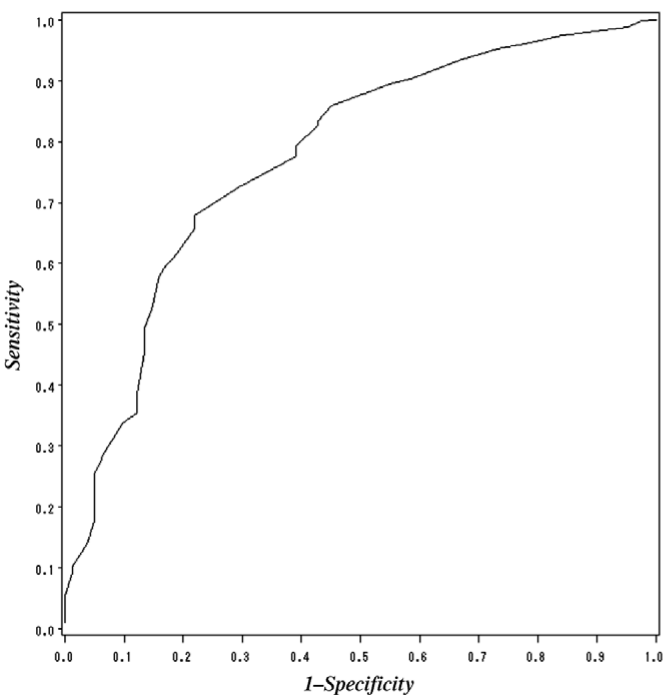

Diet plus pravastatin group

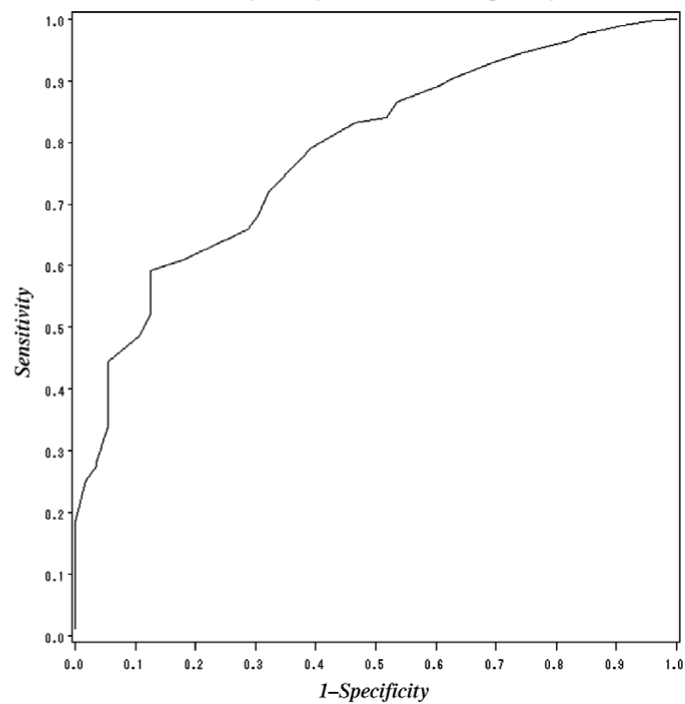

Fig 3. Receiver operating characteristic curves for the diet group and diet plus pravastatin group.

score 9) and their combination (risk score 17) compared to the scores for men (risk score 7 ), age $\geq 65$ years (risk score 8 ), and low HDL-C (risk score 6).

Notably, the risk scores for a high LDL-C (risk score 2) and smoking (risk score 3) were lower than those for the other risk factors. The risk quintiles were determined by the intrinsic cut-off points of 10, 16, 22 and 27.

Table 3 shows patients' background factors as classified by risk level. Mean age tended to be higher for the higher risk levels. There were more women than men in the lower 

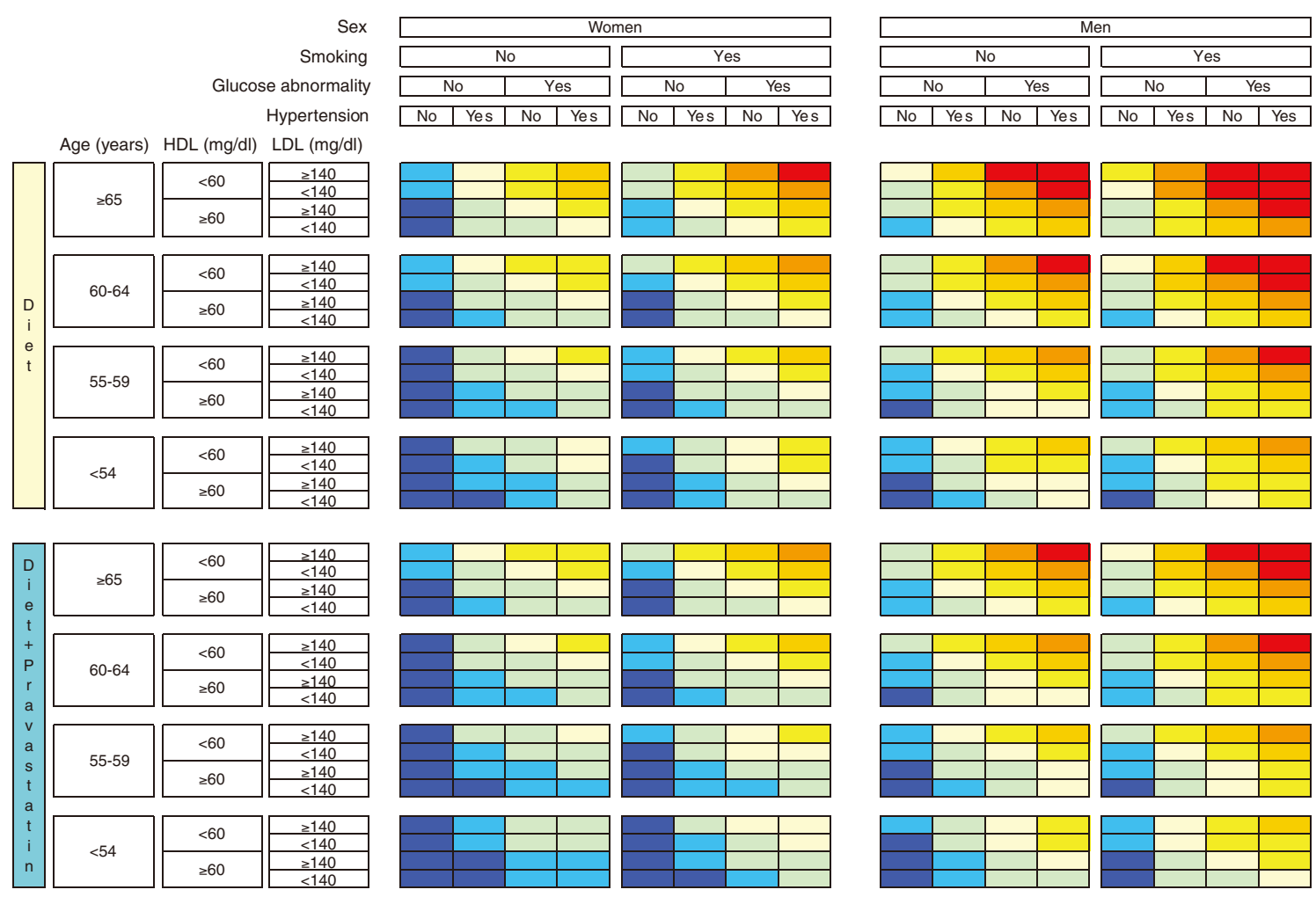

Fig 4. Risk assessment chart for 5-year risk of coronary heart disease (CHD) incidence, including sex, age, high-density lipoprotein (HDL)-cholesterol, low-density lipoprotein (LDL)-cholesterol, glucose abnormality (including impaired fasting glucose), hypertension, and smoking.

risk levels. Body mass index increased (ranging from 0.26 to 0.91 ) for each increase of 1 risk level. Prevalence of glucose abnormality (documented diabetes and high fasting blood sugar concentration), hypertension, and smoking habit were greater in the higher risk levels, whereas TC concentration was similar across risk levels. LDL-C concentrations were similar for the 4 highest levels of risk and were somewhat lower for the lowest risk level. HDL-C concentrations were higher at the lower levels of risk and tended to decrease as the level of risk increased. TG tended to increase slightly as the risk levels increased, whereas concentrations of $\mathrm{Lp}$ (a) tended to decrease slightly as the risk levels increased.

The specificity of the MEGA risk assessment was validated with good concordance between the 5-year predicted values and the observed incidence. The range of the 5-year predicted values was similar for the predicted values (ranging from $0.3 \%$ to $6.4 \%$ diet group, $0.2 \%$ to $4.5 \%$ diet plus pravastatin group) and the observed values ( $0.6 \%$ to $6.3 \%$ diet group, 0 to $4.4 \%$ diet plus pravastatin group, Fig 2). The area under the curve (AUC) for the ROC curves was 0.774 for the diet group and 0.784 for the diet plus pravastatin group (Fig 3 ).

The MEGA risk prediction chart was constructed as shown in Fig 4. It depicts the increasing risk with its 8-color grading, according to the combination of risk factors in men and women in each treatment group. The 5-year predicted risk was higher in the diet group than in the diet plus pravastatin group. A 5 -year CHD risk $\geq 10 \%$ was found for men $>65$ years old and men 60-64 years old who were smokers and diabetic in the diet group (red cells), and for women $>65$ years old who were smokers and had glucose abnormality plus hypertension. When all the risk factors were present, the highest predicted risk was estimated at $21 \%$ for men and at $10 \%$ for women $<65$ years old in the diet group (data not shown).

\section{Discussion}

The MEGA risk prediction score and MEGA risk prediction chart reported here are CHD risk assessment tools developed using MEGA study data. The MEGA risk score easily predicts 5 grades of 5-year CHD risk, and even greater accuracy of risk prediction is achieved with the MEGA risk chart with 8 grades of risk, in mild to moderate hypercholesterolemia without a history of cardiovascular disease. The risk factors included in the MEGA risk prediction tools have been well known as CHD risk factors $6,7,22$ The accumulation of these risk factors is associated with a higher CHD risk,$^{14}$ and the MEGA risk tools are consistent with these findings.

The MEGA Study included patients with hypercholester- 
olemia who were 40 to 70 years old (postmenopausal women $<70$ years) with a TC concentration of $220-270 \mathrm{mg} / \mathrm{dl}$ and no past history of cardiovascular disease. These patients were recruited from outpatient clinics, therefore, the MEGA risk tools are useful to predict the likelihood of developing CHD over 5 years in typical people with mild to moderate hypercholesterolemia with no history of cardiovascular disease. A key characteristic of the MEGA risk tools is that it is possible to assess risk in people treated with diet alone and in people receiving pharmacotherapy. Notably, the Framingham risk score did not predict risk accurately in patients treated with pravastatin in a WOSCOPS substudy! ${ }^{16}$

Interestingly, an exponential increase in the predicted value was found as the risk levels increased. A near doubling in the 5-year predicted value from risk level 1 to risk level 2 was found with the MEGA risk prediction score. An even greater increase in predicted value was seen when risk was increased from level 4 to level 5.

Notably, little difference was seen between the estimated and observed incidence of CHD in both treatment groups with the MEGA risk score. This is consistent with what would be expected, when considering the association between the distribution of cases with risk factors and the increasing CHD risk across risk levels (Table 2).

The AUC of the ROC curve plotted in terms of total risk score for each treatment group was higher than 0.77 in both treatment groups. In a study reported previously, the AUC was 0.76 for the prediction of the main cardiovascular events (fatal and non-fatal myocardial infarction, coronary insufficiency [prolonged angina with documented electrocardiographic changes], heart failure, and stroke) using the Framingham risk model 23 Thus, the correspondence between these AUC values indicate the CHD risk predicted by the MEGA risk prediction score has a precision similar to that by the Framingham risk model.

A lower 5-year predicted risk was obtained with the MEGA risk prediction score than with the Framingham risk model. For the diet only treatment group, the predicted risk was one-sixth lower in the low-risk level and two-thirds lower in the high-risk level with the MEGA risk score compared with the Framingham model. A simple comparison of the 2 prediction models might not be possible because the Framingham model was developed based on a general population without left ventricular hypertrophy $(\mathrm{LVH})$, whereas the MEGA Study included patients with LVH. Further, it has been reported that the Framingham risk model is not applicable in different populations. 24,25 Thus, the MEGA risk score might be superior in its accuracy for determining CHD risk in patients with moderate hypercholesterolemia, such as Japanese patients.

The MEGA risk prediction chart provides even greater accuracy because of the incorporation of multiple risk factors. The 8 levels of risk predicted are color-coded, with the 5-year risk increasing from the bottom-left to upper-right of the chart, according to sex and age in combination with lipid factors and smoking, glucose abnormality, and hypertension. Concordance between the MEGA risk score and the MEGA risk chart is validated by the use of the same analysis model for both, with a different $\beta$ coefficiency used for the risk chart to account for it having 1 less category of HDL-C and LDL-C than the risk score.

In the present study, 2 types of risk prediction tools were developed that apply to each treatment group. As noted previously, the Framingham risk model has been shown to underestimate the risk in patients who are treated with a statin. A substudy of WOSCOPS, a primary prevention study similar to our study, calculated CHD risk using the Framingham risk model and compared the observed incidence using time course changes in mean concentrations of serum cholesterol and HDL-C ${ }^{15}$ In the placebo group the observed incidence and predicted risk were similar, whereas in the pravastatin group the observed incidence was lower than the predicted risk. Thus, the efficacy of pravastatin to reduce CHD risk is not sufficiently explained by changes in serum lipid concentrations, based on the Framingham risk model. It seems, therefore, that CHD risk should be calculated separately based on treatment or not with a statin.

There are a few limitations to our analyses. First, the MEGA risk prediction tools are applicable to patients with mild to moderate hypercholesterolemia (TC 220-270 mg/dl). Notably, however, $70 \%$ of the estimated 20 million ambulatory patients with hypercholesterolemia in Japan fall within this range. Second, the MEGA prediction tools are applicable to treated patients only, including diet treatment, as it is based on data from patients treated in the MEGA study. The predicted CHD risk for untreated persons using the MEGA risk tools is likely to be lower than the actual risk. Third, these risk prediction tools are based on data in Japanese patients, although it is feasible to consider using these tools in people with a similar profile.

We believe the MEGA risk prediction tools are valuable for use in usual clinical practice, with greater ease and accuracy to predict the 5-year CHD risk in patients with mild to moderate hypercholesterolemic patients, such as Japanese patients. Moreover, these are highly useful as educational tools for high-risk patients.

\section{Acknowledgment}

This study was financially supported by the Japanese Ministry of Health, Labor and Welfare for the first 2 years of the study, and thereafter the study was funded by Daiichi-Sankyo Co, Ltd, Tokyo, Japan.

\section{References}

1. American Heart Association. Heart disease and stroke statistics: 2006 update. Dallas: American Heart Association, 2006.

2. Health and Welfare Statistics Association. Statistical Abstracts on Health and Welfare in Japan 2006. Tokyo: Health and Welfare Statistics Association, 2007.

3. Kannel WB, Dawber TR, Kagen A, Revostskie N, Stokes J 3rd. Factors of risk in the development of coronary heart disease-six year follow-up experience: The Framingham Study. Ann Intern Med 1961; 55: 33-50.

4. Satoh H, Nishino T, Tomita K, Saijo Y, Kishi R, Tsutsui H. Risk factors and the incidence of coronary artery disease in young middleaged Japanese men: Results from a 10-year cohort study. Intern Med 2006; 45: 235-239.

5. Okamura T, Nakamura K, Kanda H, Hayakawa T, Hozawa A, Murakami Y, et al; Health Promotion Research Committee, Shiga National Health Insurance Organizations. Effect of combined cardiovascular risk factors on individual and population medical expenditures: A 10-year cohort study of national health insurance in a Japanese population. Circ J 2007; 71: 807-813.

6. Jousilahti P, Vartiainen E, Tuomilehto J, Puska P. Sex, age, cardiovascular risk factors, and coronary heart disease: A prospective follow-up study of 14786 middle-aged men and women in Finland. Circulation 1999; 99: 1165-1172.

7. The West of Scotland Coronary Prevention Study Group, Cobbe SM. Baseline risk factors and their association with outcome in the West of Scotland Coronary Prevention Study: The West of Scotland Coronary Prevention Study Group. Am J Cardiol 1997; 79: 756-762.

8. Wilson PW, D'Agostino RB, Levy D, Belanger AM, Silbershatz H, Kannel WB. Prediction of coronary heart disease using risk factor categories. Circulation 1998; 97: 1837-1847.

9. Knuiman MW, Vu HT. Prediction of coronary heart disease mortality in Busselton, Western Australia: An evaluation of the Framingham 
national health epidemiologic follow up study, and WHO ERICA risk scores. J Epidemiol Community Health 1997; 51: 515-519.

10. Thomsen TF, Davidsen M, Ibsen H, Jorgensen T, Jensen G, BorchJohnsen K. A new method for CHD prediction and prevention based on regional risk scores and randomized clinical trials; PRECARD and the Copenhagen Risk Score. J Cardiovasc Risk 2001; 8: 291-297.

11. Conroy RM, Pyorala K, Fitzgerald AP, Sans S, Menotti A, De Backer $\mathrm{G}$, et al. Estimation of ten-year risk of fatal cardiovascular disease in Europe: The SCORE project. Eur Heart J 2003; 24: 987-1003.

12. Hippisley-Cox J, Coupland C, Vinogradova Y, Robson J, May M, Brindle P. Derivation and validation of QRISK, a new cardiovascular disease risk score for the United Kingdom: Prospective open cohort study. BMJ 2007; 335: 136-141.

13. NIPPON DATA80 Research Group. Risk assessment chart for death from cardiovascular disease based on a 19-year follow-up study of a Japanese representative population. Circ J 2006; 70: 1249-1255.

14. Nakamura Y, Yamamoto T, Okamura T, Kadowaki T, Hayakawa T, Kita Y, et al; The NIPPON DATA 80 Research Group. Combined cardiovascular risk factors and outcome: NIPPON DATA80, 1980 1994. Circ J 2006; 70: 960-964.

15. Shepherd J, Cobbe SM, Ford I, Isles CG, Lorimer AR, MacFarlane $\mathrm{PW}$, et al. Prevention of coronary heart disease with pravastatin in men with hypercholesterolemia. N Engl J Med 1995; 333: 1301 1307.

16. West of Scotland Coronary Prevention Study Group. Influence of pravastatin and plasma lipids on clinical events in the West of Scotland Coronary Prevention Study (WOSCOPS). Circulation 1998; 97: $1440-1445$.

17. Management of Elevated Cholesterol in the Primary Prevention Group of Adult Japanese (MEGA) Study Group. Design and baseline characteristics of a study of primary prevention of coronary events with pravastatin among Japanese with mildly elevated cholesterol levels. Circ J 2004; 68: 860-867.

18. Nakamura H, Arakawa K, Itakura H, Kitabatake A, Goto Y, Toyota
T, et al; MEGA Study Group. Primary prevention of cardiovascular disease in Japan: Results of the management of elevated cholesterol in the primary prevention group of adult Japanese (MEGA), randomised study with pravastatin. Lancet 2006; 368: 1155-1163.

19. Friedewald WT, Levy RI, Fredrickson DS. Estimation of the concentration of low-density lipoprotein cholesterol in plasma, without use of the preparative ultracentrifuge. Clin Chem 1972; 18: 499-502.

20. Teramoto T, Sasaki J, Ueshima H, Egusa G, Kinoshita M, Shimamoto $\mathrm{K}$, et al. Executive Summary of Japan Atherosclerosis Society (JAS) Guideline for Diagnosis and Prevention of Atherosclerotic Cardiovascular Diseases for Japanese. J Atheroscler Thromb 2007; 14: 4550 .

21. Kalbfleisch JD, Prentice RL. The statistical analysis of failure time data, 2nd edn. New York: John Wiley \& Sons; 2002.

22. Yamamoto A, Richie G, Nakamura H, Hosoda S, Nobuyoshi M, Matsuzaki M, et al; ASPAC study members in Japan; Research Committee on Serum Lipid Survey 1990 Japan. Risk factors for coronary heart disease in the Japanese-comparison of the background of patients with acute coronary syndrome in the ASPAC study with data obtained from the general population: Asia-Pacific Collaboration on CHD Risk Factor Intervention study. J Atheroscler Thromb 2002; 9: 191-199.

23. Wang TJ, Gona P, Larson MG, Tofler GH, Levy D, Newton-Cheh C, et al. Multiple biomarkers for the prediction of first major cardiovascular events and death. $N$ Engl J Med 2006; 355: 2631-2639.

24. de Visser CL, Bilo HJ, Thomsen TF, Groenier KH, Meyboom-de Jong B. Prediction of coronary heart disease: A comparison between the Copenhagen risk score and the Framingham risk score applied to a Dutch population. J Intern Med 2003; 253: 553-562.

25. Neuhauser HK, Ellert U, Kurth BM. A comparison of Framingham and SCORE-based cardiovascular risk estimates in participants of the German National Health Interview and Examination Survey 1998. Eur J Cardiovasc Prev Rehabil 2005; 12: 442-450. 